\title{
The Impact of Genomics on the Management of Myeloma
}

\author{
Jill Corre, $\mathrm{MD},{ }^{\mathrm{a}}$ and Hervé Avet-Loiseau, MD, $\mathrm{PhD},{ }^{\mathrm{b}}$ Toulouse and Nantes, France
}

Key Words

Myeloma, genomics, prognosis

\begin{abstract}
Myeloma is a complex disease, characterized by a wide heterogeneity in clinical presentation, evolution, and molecular portraits. The successive use of cytogenetics, molecular cytogenetics, expression genomics, copy number genomics, and, more recently, deep sequencing, has shown that this heterogeneity can be used to identify markers usable for not only prognostication but also therapeutic choice and, ultimately, discovery of druggable targets. The use of some of these techniques is now mandatory for the management of patients. Although risk-adapted therapy is not yet a routine practice in myeloma, these molecular changes are essential for the definition of the prognosis. (JNCCN 2011;9:1200-1207)
\end{abstract}

\section{Medscape: Continuing Medical Education Online}

\section{Accreditation Statement}

This activity has been planned and implemented in accordance with the Essential Areas and policies of the Accreditation Coun- cil for Continuing Medical Education through the joint sponsorship of Medscape, LLC and JNCCN - The Journal of the National Comprehensive Cancer Network. Medscape, LLC is accredited by the ACCME to provide continuing medical education for physicians.

$\begin{array}{ll}\text { Medscape } & \text { Medscape, LLC designates this Journal-based CME } \\ \text { activity for a maximum of } 1.0 \text { AMA PRA Cate- } & \text { gory } 1 \text { Credit(s) } \text { TM. Physicians should claim only }^{\text {and }}\end{array}$
the credit commensurate with the extent of their participation in the activity.

All other clinicians completing this activity will be issued a certificate of participation. To participate in this journal CME activity: (1) review the learning objectives and author disclosures; (2) study the education content; (3) take the post-test with a $70 \%$ minimum passing score and complete the evaluation at www.medscape. org/journal/jnccn; (4) view/print certificate.

Release date: October 7, 2011; Expiration date: October 7, 2012.

\section{Learning Objectives}

Upon completion of this activity, participants will be able to:

- Distinguish the most common genetic abnormality in multiple myeloma

- Analyze how specific genetic abnormalities affect the prognosis of multiple myeloma

- Evaluate how genetic findings in multiple myeloma can affect the choice of treatment
From ${ }^{a}$ Centre Hospitalier Universitaire, Université, UMR 1037, Toulouse


Nantes, France.

Submitted April 26, 2011; accepted for publication June 28, 2011.

Correspondence: Hervé Avet-Loiseau, MD, PhD, Laboratoire

d'Hématologie, Institut de Biologie, 9 quai Moncousu, 44093 Nantes

Cedex 1, France.

E-mail: havetloiseau@chu-nantes.fr

\section{EDITOR}

Kerrin M. Green, MA, Assistant Managing Editor, Journal of the National Comprehensive Cancer Network

Disclosure: Kerrin M. Green, MA, has disclosed no relevant financial relationships.

\section{AUTHORS AND CREDENTIALS}

Jill Corre, MD, Centre Hospitalier Universitaire, Université, Toulouse, France

Disclosure: Jill Corre, MD, has disclosed no relevant financial relationships.

Hervé Avet-Loiseau, MD, PhD, Centre Hospitalier Universitaire, Université, Nantes, France.

Disclosure: Hervé Avet-Loiseau, MD, PhD, has disclosed no relevant financial relationships.

\section{CME AUTHOR}

Charles P. Vega, MD, Health Sciences Clinical Professor; Residency Director, Department of Family Medicine, University of California, Irvine Disclosure: Charles P. Vega, MD, has disclosed no relevant financial relationships. 
Impact of Genomics in Myeloma Management

Despite major improvements in the prognosis of multiple myeloma in the past decade (mainly because of the availability of novel drugs, such as thalidomide, bortezomib, and lenalidomide), patient outcomes are highly heterogeneous. Even though the median survival of the youngest patients is probably now more than 10 years, some patients are still presenting with refractory disease, with a fatal evolution within weeks or months. Many factors can drive this evolution. Apart from patient-related factors, such as comorbidities (including poor performance status and cardiac, renal, or liver dysfunctions), the most important heterogeneity is related to intrinsic malignant plasma cell variations. The basis of this heterogeneity is probably within the wide spectrum of molecular rearrangements observed in the malignant plasma cells. During the past decade, many studies exploring genetic rearrangements have been published. This article addresses the abnormalities that could be used in the management of patients with myeloma.

\section{Pathogenesis}

Although karyotypic complexity is the rule in myeloma, recurrent changes have been identified. ${ }^{1-5}$ Briefly, hyperdiploidy (with recurrent chromosomal gains) is observed in $50 \%$ to $60 \%$ of patients, whereas monosomy 13 is seen in $45 \%$. Recurrent structural rearrangements are also observed, targeting especially the IGH gene mapped at $14 \mathrm{q} 32$. The karyotypic oncogenetic classification, mainly based on hyperdiploidy and $14 \mathrm{q} 32$ translocations, ${ }^{6}$ is only partially confirmed by molecular studies. However, several studies have evaluated myeloma at the transcriptional level using gene expression profiling. With this approach, CD138+ purified malignant plasma cells are used to extract RNAs and hybridized on arrays to evaluate the expression of genes representative of the whole transcribed genome. Using unsupervised bioinformatic methods, tumors are then classified according to gene expression profile similarities.

One of the first analyses compared gene expression profiling in cohorts of patients with monoclonal gammopathy of undetermined significance (MGUS) and myeloma, showing sequential genetic changes from normal plasma cells to malignant plasma cells in the process, providing clues to a molecular basis for malignant transformation and potential therapeutic targets. ${ }^{7}$ The first molecular classification in 2002 clustered genes according to similarities with either MGUS or human myeloma cell lines, ${ }^{8}$ and identified 4 classes of myelomas according to these similarities.

In 2003, another study showed that the most relevant profiles were related to immunoglobulin (Ig) gene expression. ${ }^{9}$ More recently, using unsupervised analyses, 3 reports identified subgroups mostly driven by chromosomal aberrations. The first report identified 8 different subgroups, mainly based on the cyclin D gene expression and the different $14 \mathrm{q} 32$ recurrent translocations. ${ }^{6}$ This molecular classification was refined in 2006, identifying 7 subclasses of myeloma. ${ }^{10}$ In this pathogenetic model, the first class is defined by the translocation $\mathrm{t}(4 ; 14)$, identified by overexpression of the MMSET or FGFR3 genes. The second class is defined by upregulation of one of the MAF genes, related to the translocations $t(14 ; 16)$ or $\mathrm{t}(14 ; 20)$. Cases with CCND1 or CCND3 upregulation (from the translocations $\mathrm{t}(11 ; 14)$ or $\mathrm{t}(6 ; 14)$ ) clustered in 2 different groups, and are named CD1 and CD2. The CD2 group was characterized by CD20 expression. The fifth group was characterized by hyperdiploidy. The last 2 groups were characterized by a low incidence of bone disease, according to a low DKK1 expression, whereas the last group was characterized by a high expression of genes involved in proliferation. This molecular classification has been partially confirmed and further refined by a recent study by the HOVON group. ${ }^{11}$ Although the "low bone disease" group was not confirmed, 3 other subgroups were identified: one enriched by myeloid genes (that could be related to plasma cell sorting problems), one characterized by overexpression of cancer-testis antigen genes, and finally one defined by overexpression of positive regulators of the nuclear factor $\kappa \mathrm{B}(\mathrm{NF}-\kappa \mathrm{B})$ pathway.

DNA-based techniques, such as array comparative genomic hybridization, ${ }^{12,13}$ have identified the role of the NF- $\mathrm{KB}$ pathway in myeloma. Two separate studies have shown that the NF- $\kappa B$ pathway can be activated, either through deletions of NF- $\mathrm{KB}$ inhibitors (such as TRAF3, BIRC, or CYLD) or through activation of NF- $\mathrm{KB}$ activators (such as NIK or CD40). Other studies based on the analysis of copy number changes using high-density single nucleotide polymorphism (SNP) arrays have identified other levels of molecular heterogeneity. ${ }^{14-17}$ These studies identified genomic heterogeneity within the hyperdiploid group driven by the presence of either chromosome 1q and/ 
or 11 gain, chromosome 13 loss, or chromosome 5 gain, conferring a significant outcome difference. Furthermore, these studies showed that integration of copy number changes and gene expression values allowed the conversion of genomic heterogeneity into identification of potential cancer target genes. Subclasses of hyperdiploid patients with multiple myeloma with clinical and biologic associations were also characterized using gene expression profiling. ${ }^{18}$ Initial attempts at understanding the genesis of these genomic heterogeneities were based on uncontrolled recombination mechanisms, which may become a potential target for understanding the biology and developing an effective therapeutic strategy. ${ }^{19}$

\section{Implications for Prognosis}

If molecular studies have not yet provided a definite myeloma subclassification into specific diseases correlating with biology or clinical behavior, they have definitely contributed to the identification of several prognostic factors that significantly influence patient outcomes. Conventional cytogenetics have identified recurrent chromosomal changes and correlated these with clinical outcomes (Table 1). Abnormalities such as $t(4 ; 14)$ and $t(14 ; 16)$, and loss of $17 \mathrm{p} 13$ confer a poor prognosis in patients undergoing high-dose therapy. In contrast, hyperdiploidy has been associated with better outcomes, even though it is a heterogeneous category. Because myeloma cells have a low proliferative index, the prognostic significance of genetic abnormalities is analyzed with interphase fluorescence in situ hybridization (FISH). However, these FISH analyses require plasma cell recognition or sorting.

Among the specific $14 \mathrm{q} 32$ translocations discussed previously, $\mathrm{t}(4 ; 14)$ is definitely the most important one from a clinical standpoint. Several studies have confirmed that patients who display this translocation $(15 \%)$ have a specifically poor prognosis. ${ }^{20-25}$ These patients may require a specific therapeutic approach to include the novel agents such as proteasome inhibitors. Previously reported monosomy 13 is not considered to predict poor outcome by itself. The most important chromosomal change for prognosis is del(17p). Present in $8 \%$ to $10 \%$ of patients, this deletion is associated with a remarkably short survival, irrespective of the therapy used. ${ }^{24,26,27}$ The molecular target of this deletion could be TP53, but no clear biologic evidence supports this hypothesis, and mu- tations are observed in only a subset of patients with $\operatorname{del}(17 p) .{ }^{28}$ Finally, several reports have shown that gains of chromosome 1q (observed in one-third of the patients) also confer a poor prognosis..$^{29,30}$ This abnormality is typically a secondary event, not specific of myeloma, acquired during evolution.

The prognostic significance of molecular changes have been analyzed using high-throughput microarray profiling techniques, focused on copy number alteration using either SNP array or gene expression profiling. These techniques are potentially more powerful because they analyze the whole genome. An analysis of genome-wide copy number alterations (CNA) in 192 uniformly treated patients with newly diagnosed myeloma using high-density SNP arrays suggested a global genomic instability in myeloma. ${ }^{15}$ One of 3 distinct patterns of CNAs are present in $98 \%$ of cases: loss or gain of the chromosome, loss or gain of a whole arm of the chromosome, or interstitial losses or gains. Analyses of the most frequent lesions (> 10\%) identified 2 main groups: the first group encompasses almost exclusively (except for chromosome 11) either gain or loss of entire chromosomes, or interstitial gain or loss of the flanking centromeric regions. This group includes gains of chromosomes 3, 5, 7, 9, 11, 15, 19, and 21 and loss of chromosomes 13, 22, and X (in female cases). The second group is characterized by genetic lesions that affect gain or loss of subchromosomal material, including gains of $1 q$ and $6 p$ and deletions of $1 p, 6 q$, 8p, 12p, 14q, 16p, 16q, and 20p.

Analysis of the prognostic significance of $\mathrm{CNAs}$ in myeloma identified that gains of $1 \mathrm{q}$ and deletions of $1 p, 12 p, 14 q, 16 q$, and $22 q$ were associated with poor prognosis, whereas gains of chromosomes 5, 9, 11,15 , and 19 conferred a superior outcome. A multivariate analysis identified a prognostic model that includes amp(1q23.3), amp(5q31.3), and del(12p13.31) as the most powerful independent adverse markers $(P<.0001)$, and the prognostic significance of the model was validated in an independent cohort of 273 patients with myeloma. These findings therefore show the feasibility of molecular karyotyping using SNP profiling to predict outcome in myeloma. This prognostic model must be confirmed in an independent series. Recurrent cytogenetic changes in myeloma are listed in Table 1.

Two large studies have evaluated the prognostic significance of gene expression profiling in identifying poor-risk patient populations. One of the 2 mod- 
Impact of Genomics in Myeloma Management

\begin{tabular}{lll} 
Table 1 & $\begin{array}{l}\text { Recurrent Cytogenetic Changes in } \\
\text { Myeloma }\end{array}$ \\
Abnormality & Frequency & Prognosis \\
Hyperdiploidy & $50 \%-60 \%$ & $\begin{array}{l}\text { Good/neutral } \\
\mathrm{t}(4 ; 14)\end{array}$ \\
& $15 \%$ & $\begin{array}{l}\text { Poor (neutral if } \\
\text { bortezomib-based } \\
\text { therapy) }\end{array}$ \\
$\mathrm{t}(11 ; 14)$ & & Neutral \\
$\mathrm{t}(14 ; 16)$ & $20 \%$ & Poor/neutral \\
Monosomy 13 & $3 \%$ & Neutral \\
del(17p) & $45 \%$ & Poor \\
$1 \mathrm{q}$ gain & $8 \%$ & Poor \\
del(1p) & $35 \%$ & Poor \\
$5 q$ gain & $30 \%$ & Good \\
del(12p) & $50 \%$ & Poor \\
\hline
\end{tabular}

els, the University of Arkansas for Medical Science (UAMS) 70-gene model, has 30\% of the informative genes mapped to chromosome $1 .{ }^{31}$ In the other model, the Intergroupe Francophone du Myelome (IFM) 15-gene model, high-risk patients were enriched in genes controlling proliferation and chromosomal instability, whereas low-risk patients were enriched in hyperdiploid karyotypes. ${ }^{32}$

Interestingly, the 2 models do not have a single common gene, reflecting mainly the redundancy in the genes and pathways that control growth, proliferation, and survival, in addition to differences in the platforms used for the microarray analyses or in the treatment used to define the patient population. However, in an attempt to validate the techniques, the IFM 15-gene set was shown to be powerful in the UAMS population, but with a lower significance. ${ }^{32}$ Interestingly, both sets identified patients with a short survival but neither identified very good-risk patients, probably because of a short follow-up. An international large-scale effort is needed to fully validate a uniform set of genes predictive of outcome, irrespective of the treatment used, to make gene expression profiling routine in clinical practice.

Moreover, because $\mathrm{CpG}$ methylation affects gene expression and thus may be relevant to pathogenesis and behavior of myeloma cells, a genomewide methylation profile has been analyzed using microarray. A recent study showed that methylation patterns, especially hypomethylation, were capable of distinguishing nonmalignant from malignant plasma cells. ${ }^{33}$ In fact, differential methylation was also evident at transition of MGUS cells to myeloma cells. Interestingly, genes involved in cell-to-cell signaling and cell adhesion were remethylated in cells from the plasma cell leukemia stage, suggesting development of independence from the interaction with bone marrow microenvironment cells.

Recently, 2 transcriptome modifiers were investigated in myeloma. Alternate splicing is an important posttranslational change that alters specificity of gene function. Dysregulated alternative splicing has been reported in myeloma with an effect on overall clinical outcome. ${ }^{34}$ MicroRNA are small noncoding RNA molecules that regulate multiple target genes through cleavage of targeted transcripts and inducing translational inhibition. The differential expression of several microRNAs has been described in myeloma and MGUS compared with normal plasma cells..$^{35}$ In one study, miR-21, miR-106b 25, and miR-181 $a$ and $b$ were overexpressed in myeloma and MGUS with respect to normal plasma cells, whereas miR-32 and miR-17 92 were exclusively overexpressed in myeloma compared with MGUS. Two target genes of overexpressed miRs, SOCS-1 and p300-CBP, were identified as having influence on myeloma pathogenesis. Down-regulation of miR-15a and miR-16 present on chromosome 13 has also been described as having a potential effect on myeloma cell proliferation ${ }^{36}$; however, its relation with chromosome 13 or $13 \mathrm{q} 34$ deletion is not established. ${ }^{37}$

Some relation between $\mathrm{miR}$ expression pattern and molecular and genetic subgroups in myeloma has been described. ${ }^{38,39}$ The overexpression of let$7 e$, miR-125-5p, and miR-99b located at 19q13.33 in patients with $\mathrm{t}(4 ; 14)$ translocation, ${ }^{38}$ and $m i R-1$ and miR-133a in $\mathrm{t}(14 ; 16)$ myeloma has been reported. ${ }^{39}$ Combined mRNA and miR profiling has identified a microRNA/mRNA regulatory network with early evidence of differential expression in high-risk disease..$^{40}$ Evidence shows that miR-192, miR-194, and miR-215, which are downregulated in subsets of patients with myeloma, are correlated with transcriptional activation by $p 53$ and modulation of MDM2 expression, suggesting that these miRNAs are positive regulators of $p 53$ with an important role in myeloma development. ${ }^{41}$ Unsupervised clustering analysis of microRNA expression profile data also identifies groups with different survival outcomes, recognizing critical microRNAs as modulators of gene expression and signaling pathways, and pro- 
vides potential novel microRNA and gene targets in myeloma for both understanding of biological behavior and therapeutic application. ${ }^{42}$

\section{Implications for Patient Management}

Treatment options have been especially driven by age or physiologic conditions. In patients younger than 65 years, the standard of care is usually a short induction (including novel drugs) followed by high-dose melphalan with stem cell rescue. For older patients or those with comorbidities, long-term treatment with a combination is usually chosen. However, the availability and understanding of genomic data have significantly contributed to the treatment of myeloma. Use of genetic data in treatment selection has been proposed based on patients' myeloma cell genetic characteristics, such as in those displaying the $t(4 ; 14)$. A few studies showed that patients with $t(4 ; 14)$ may benefit from the use of bortezomib as either induction therapy or long-term treatment. ${ }^{43-45}$ In some of these studies, the long-term use of bortezomib totally overcame the poor prognosis associated with $\mathrm{t}(4 ; 14) .43,44$

For other high-risk parameters, such as $\operatorname{del}(17 \mathrm{p})$ or gene expression-defined high-risk disease, no specific treatment has shown a beneficial effect. Another important aspect would be to define a standard of care for very good-risk patients. However, these patients are not yet clearly identified, and long-term analyses are needed to define these patients and then to possibly propose less-toxic approaches to their treatment. A recent analysis of patients treated with VAD (vincristine, adriamycin, dexamethasone) induction followed by a double course of high-dose melphalan showed that patients younger than 55 years with a $\beta_{2}$ microglobulin level less than $5.5 \mathrm{mg} / \mathrm{L}$, lacking both $\mathrm{t}(4 ; 14) \operatorname{del}(17 \mathrm{p})$, and with a $1 \mathrm{q}$ gain had an overall survival of $75 \%$ at 8 years. ${ }^{46}$ Thus, genetic/genomic analyses also seem to be useful in identifying patients with long survival expectancy. Finally, a major objective for individualized therapeutic approaches would be to define what is the best frontline or subsequentline combination treatment for a specific patient. This objective requires genomic studies performed in welldefined populations of patients, treated with a specific combination (such as bortezomib-dexamethasone or lenalidomide-dexamethasone), with a primary end point based on progression-free survival. Several studies are ongoing.

\section{Next-Generation Sequencing}

Recently, 2 reports presented data on sequencing in myeloma. The first study used massively parallel whole-genome paired-end sequencing on 2 myeloma patient samples collected 6 months apart and identified 29 somatic rearrangements, including 3 that were present only in the second sample. ${ }^{47}$ One of these was on chromosome 13. Breakpoint sequencing showed a $64.9 \mathrm{kB}$ homozygous (no wild-type read pairs found) deletion involving the first 2 exons of the RB1 gene. No reads spanning this breakpoint were found in the matching sample taken 6 months earlier. A second much larger effort in 29 patients (22 whole genomes and 17 whole exomes) using $30 x$-coverage deep sequencing identified several unique recurrent biologically important mutations involving histone methyltransferases, transcription factor IRF4, BRAF, genes involved in protein translation, genes involved in the NF- $\mathrm{KB}$ pathway, and, surprisingly, genes involved in blood coagulation. ${ }^{48}$ These early sequencing efforts provide important insight into the pathogenesis of disease progression and confirm the potential of whole-genome sequencing to inform on the biology of the disease, which may affect the therapeutic approach in future.

\section{Conclusions}

All of the reported studies show that myeloma is characterized by a wide molecular heterogeneity. The next steps will be to develop a combination of several molecular approaches, including copy number change analyses, gene expression profiling, massive parallel sequencing, miRNA analyses, and epigenetic changes survey in large uniformly treated patient cohorts. This will provide a clear landscape of the molecular changes and their impact on myeloma classification, prognosis, and, ultimately, therapeutic management.

\section{References}

1. Dewald GW, Kyle RA, Hicks GA, Greipp PR. The clinical significance of cytogenetic studies in 100 patients with multiple myeloma, plasma cell leukemia, or amyloidosis. Blood 1985;66:380390.

2. Sawyer JR, Waldron JA, Jagannath S, Barlogie B. Cytogenetic finding in 200 patients with multiple myeloma. Cancer Genet Cytogenet 1995;82:41-49.

3. Laï JL, Zandecki M, Mary JY, et al. Improved cytogenetics in 
Impact of Genomics in Myeloma Management

multiple myeloma: a study of 151 patients including 117 patients at diagnosis. Blood 1995;85:2490-2497.

4. Calasanz MJ, Cigudosa JC, Odero MD, et al. Cytogenetic analysis of 280 patients with multiple myeloma and related disorders: primary breakpoints and clinical correlations. Genes Chromosom Cancer 1997;18:84-93.

5. Smadja NV, Bastard C, Brigaudeau C, et al. Hypodiploidy is a major prognostic factor in multiple myeloma. Blood 2001;98:2229-2238.

6. Bergsagel PL, Kuehl WM, Zhan F, et al. Cyclin D dysregulation: an early and unifying pathogenic event in multiple myeloma. Blood 2005;106:296-303.

7. Davies FE, Dring AM, Li C, et al. Insights into the multistep transformation of MGUS to myeloma using microarray expression analysis. Blood 2003;102:4504-4511.

8. Zhan F, Hardin J, Kordsmeier B, et al. Global gene expression profiling of multiple myeloma, monoclonal gammopathy of undetermined significance, and normal bone marrow plasma cells. Blood 2002;99:1745-1757.

9. Magrangeas F, Nasser V, Avet-Loiseau H, et al. Gene expression profiling of multiple myeloma reveals molecular portraits in relation to the pathogenesis of the disease. Blood 2003;101:4998-5006.

10. Zhan F, Huang Y, Colla S, et al. The molecular classification of multiple myeloma Blood 2006;108:2020-2028.

11. Broyl A, Hose D, Lokhorst $\mathrm{H}$, et al. Gene expression profiling for molecular classification of multiple myeloma in newly diagnosed patients. Blood 2010;116:2543-2553.

12. Annunziata CM, Davis RE, Demchenko $\mathrm{Y}$, et al. Frequent engagement of the classical and alternative NFKB pathways by diverse genetic abnormalities in multiple myeloma. Cancer Cell 2007;12:115-130.

13. Keats JJ, Fonseca R, Chesi M, et al. Promiscuous mutations activate the noncanonical NFKB pathway in multiple myeloma. Cancer Cell 2007;12:131-144.

14. Carrasco DR, Tonon G, Huang Y, et al. High-resolution genomic profiles define distinct clinicopathogenetic subgroups of multiple myeloma patients. Cancer Cell 2006;9:313-325.

15. Avet-Loiseau H, Li C, Magrangeas F, et al. Prognostic significance of copy-number alterations in multiple myeloma. J Clin Oncol 2009;27:4585-4590.

16. Fonseca R, Bergsagel PL, Drach J, et al. International Myeloma Working Group. International Myeloma Working Group molecular classification of multiple myeloma: spotlight review. Leukemia 2009;23:2210-2221.

17. Walker BA, Leone PE, Chiecchio L, et al. A compendium of myeloma-associated chromosomal copy number abnormalities and their prognostic value. Blood 2010;116:56-65.

18. Chng WJ, Kumar S, Vanwier S, et al. Molecular dissection of hyperdiploid multiple myeloma by gene expression profiling. Cancer Res 2007;67:2982-2989.

19. Shammas MA, Shmookler Reis RJ, Koley H, et al. Dysfunctional homologous recombination mediates genomic instability and progression in myeloma. Blood 2009;113:2290-2297.

20. Chang H, Sloan S, Li D, et al. The $\mathrm{t}(4 ; 14)$ is associated with poor prognosis in myeloma patients undergoing autologous stem cell transplant. Br J Haematol 2004;125:64-68.

21. Gutierrez NC, Castellanos MV, Martin ML, et al. Prognostic and biological implications of genetic abnormalities in multiple myeloma undergoing autologous stem cell transplantation: $t(4 ; 14)$ is the most relevant adverse prognostic factor, whereas RB deletion as a unique abnormality is not associated with adverse prognosis.
Leukemia 2007;21:143-150.

22. Gertz MA, Lacy MQ, Dispenzieri A, et al. Clinical implications of $\mathrm{t}(11 ; 14)(\mathrm{q} 13 ; \mathrm{q} 32), \mathrm{t}(4 ; 14)(\mathrm{p} 16.3 ; \mathrm{q} 32)$, and $-17 \mathrm{p} 13$ in myeloma patients treated with high-dose therapy. Blood 2005;106:28372840.

23. Moreau P, Attal M, Garban F, et al. Heterogeneity of $t(4 ; 14)$ in multiple myeloma. Long-term follow-up of 100 cases treated with tandem transplantation in IFM99 trials. Leukemia 2007;21:2020 2024.

24. Avet-Loiseau H, Attal M, Moreau P, et al. Genetic abnormalities and survival in multiple myeloma: the experience of the Intergroupe Francophone du Myélome. Blood 2007;109:3489-3495.

25. Fonseca R, Blood E, Rue M, et al. Clinical and biologic implications of recurrent genomic aberrations in myeloma. Blood 2003;101:4569-4575.

26. Drach J, Ackermann J, Fritz E, et al. Presence of a p53 gene deletion in patients with multiple myeloma predicts for short survival after conventional-dose chemotherapy. Blood 1998;92:802-809.

27. Chang H, Qi C, Yi QL, et al. p53 gene deletion detected by fluorescence in situ hybridization is an adverse prognostic factor for patients with multiple myeloma following autologous stem cell transplantation. Blood 2005;105:358-360.

28. Lode L, Eveillard M, Trichet V, et al. Mutations in TP53 are exclusively associated with del(17p) in multiple myeloma. Haematologica 2010;95:1973-1976.

29. Hanamura I, Stewart JP, Huang Y, et al. Frequent gain of chromosome band 1q21 in plasma-cell dyscrasias detected by fluorescence in situ hybridization: incidence increases from MGUS to relapsed myeloma and is related to prognosis and disease progression following tandem stem-cell transplantation. Blood 2006;108:1724-1732.

30. Fonseca R, Van Wier SA, Chng WJ, et al. Prognostic value of chromosome 1q21 gain by fluorescent in situ hybridization and increase CKS1B expression in myeloma. Leukemia 2006;20:20342040.

31. Shaughnessy JD, Zhan F, Burrington BE, et al. A validated gene expression model of high-risk multiple myeloma is defined by deregulated expression of genes mapping to chromosome 1. Blood 2007;109:2276-2284.

32. Decaux O, Lode L, Magrangeas F, et al. Prediction of survival in multiple myeloma based on gene-expression profiles revealed cell cycle and chromosomal instability signatures in high-risk patients and hyperdiploid signatures in low-risk patients. J Clin Oncol 2008;26:4798-4805.

33. Walker BA, Wardell CP, Chiecchio L, et al Aberrant global methylation patterns affect the molecular pathogenesis and prognosis of multiple myeloma. Blood 2011;117:553-562.

34. Munshi NC, Li C, Minvielle S, et al. Alternate splicing is a frequent event and impacts clinical outcome in myeloma: a highdensity exon array analysis of uniformly treated newly-diagnosed myeloma patients. Blood 2008;112:498a.

35. Pichiorri F, Suh SS, Ladetto M, et al. MicroRNAs regulate critical genes associated with multiple myeloma pathogenesis. Proc Natl Acad Sci U S A 2008;105:12885-12890.

36. Roccaro AM, Sacco A, Thompson B, et al. MicroRNAs 15a and 16 regulate tumor proliferation in multiple myeloma. Blood 2009;113:6669-6680.

37. Corthals SL, Jongen-Lavrencic M, de Knegt Y, et al. Micro-RNA15a and micro-RNA-16 expression and chromosome 13 deletions in multiple myeloma. Leuk Res 2010;34:677-681. 
38. Lionetti $\mathrm{M}$, Biasiolo $\mathrm{M}$, Agnelli $\mathrm{L}$, et al. Identification of microRNA expression patterns and definition of a microRNA/ mRNA regulatory network in distinct molecular groups of multiple myeloma. Blood 2009;114:20-26.

39. Gutiérrez NC, Sarasquete ME, Misiewicz-Krzeminska I, et al. Deregulation of microRNA expression in the different genetic subtypes of multiple myeloma and correlation with gene expression profiling. Leukemia 2010;24:629-637.

40. Zhou Y, Chen L, Barlogie B, et al. High-risk myeloma is associated with global elevation of miRNAs and overexpression of EIF2C2/ AGO2. Proc Natl Acad Sci U S A 2010;107:7904-7909.

41. Pichiorri F, Suh SS, Rocci A, et al. Downregulation of p53. inducible microRNAs 192, 194, and 215 impairs the p53/MDM2 autoregulatory loop in multiple myeloma development. Cancer Cell 2010;18:367-381.

42. Adamia S, Avet-Loiseau H, Amin SD, et al. MicroRna expression profile identifies distinct clinically relevant sub-groups in multiple myeloma: novel prognostic markers and potential targets for therapy. Blood 2008;112:96a.

43. San Miguel JF, Schlag R, Khuageva NK, et al. Bortezomib plus melphalan and prednisone for initial treatment of multiple myeloma. N Engl J Med 2008;359:906-917.

44. Pineda-Roman M, Zangari M, Haessler J, et al. Sustained complete remissions in multiple myeloma linked to bortezomib in total therapy 3: comparison with total therapy 2. Br J Haematol 2008;140:625-634.

45. Avet-Loiseau H, Leleu X, Roussel M, et al. Bortezomib plus dexamethasone induction improves outcome of patients with $\mathrm{t}(4 ; 14)$ myeloma but not outcome of patients with del(17p). J Clin Oncol 2010;28:4630-4634.

46. Avet-Loiseau $\mathrm{H}$, et al. Long-term analysis of the IFM 99 trials for myeloma: cytogenetic abnormalities $(\mathrm{t}(4 ; 14)$, del(17p), 1q gains) play a major role in defining long-term survival. J Clin Oncol, in press.

47. Munshi NC, Avet-Loiseau H, Stephens $\mathrm{P}$ et al. Whole genome paired end sequencing identifies genomic evolution in myeloma. Blood 2008;112:2846a.

48. Chapman MA, Lawrence MS, Keats JJ, et al. Initial genome sequencing and analysis of multiple myeloma. Nature 2011;471:467-472. 


\section{CME Activity: Genomics in Multiple Myeloma}

To obtain credit, you should first read the journal article. After reading the article, you should be able to answer the following, related, multiple-choice questions. To complete the questions (with a minimum $70 \%$ passing score) and earn continuing medical education (CME) credit, please go to www.medscape. org/journal/jncen.

Credit cannot be obtained for tests completed on paper, although you may use the worksheet below to keep a record of your answers. You must be a registered user on Medscape.org. If you are not registered on Medscape.org, please click on the New Users: Free Registration link on the left hand side of the website to register.

Only one answer is correct for each question. Once you successfully answer all post-test questions you will be able to view and/or print your certificate. For ques- tions regarding the content of this activity, contact the accredited provider, CME@medscape.net. For technical assistance, contact CME@webmd.net.

American Medical Association's Physician's Recognition Award (AMA PRA) credits are accepted in the US as evidence of participation in CME activities. For further information on this award, please refer to http:// www.ama-assn.org/ama/pub/category/2922.html. The AMA has determined that physicians not licensed in the US who participate in this CME activity are eligible for AMA PRA Category 1 Credits $^{\mathrm{TM}}$. Through agreements that the AMA has made with agencies in some countries, AMA PRA credit may be acceptable as evidence of participation in CME activities. If you are not licensed in the U.S., please complete the questions online, print the AMA PRA CME credit certificate, and present it to your national medical association for review.
1. A 67-year-old man presents with a new diagnosis of multiple myeloma. He wants to know how he developed myeloma, and you discuss the genomics of multiple myeloma. Which of the following genetic abnormalities is most common in cases of multiple myeloma?
A. Monosomy 13
B. Hyperdiploidy
C. $\mathrm{t}(4 ; 14)$
D. $\operatorname{del}(17 \mathrm{p})$

2. The patient undergoes genetic testing. Which of the following abnormalities is associated with a better prognosis for myeloma?
A. Hyperdiploidy
B. $\mathrm{t}(4 ; 14)$
C. $\mathrm{t}(14 ; 16)$
D. Loss of $17 \mathrm{p} 13$

3. Which of the following statements about other ge- netic abnormalities and their associated prognosis in multiple myeloma is accurate?
A. del(17p) has a generally neutral effect on prognosis
B. Gains of $1 \mathrm{q}$ are associated with a good prognosis
C. $\operatorname{amp}(1 \mathrm{q} 23.3)$ is a marker of good prognosis
D. $5 q$ gain is a marker of good prognosis

4. What should you consider in regard to the interaction between genetic findings and treatment in this patient?
A. Patients with del(17p) respond particularly well to lenalidomide
B. Patients with $t(4 ; 14)$ respond particularly well to bortezomib
C. Patients with 1q gain respond particularly well to thalidomide
D. Patients with $5 \mathrm{q}$ gain respond particularly well to lenalidomide

\section{Activity Evaluation}

1. The activity supported the learning objectives. Strongly Disagree $\begin{array}{lllll}1 & 2 & 3 & 4 & 5\end{array}$

2. The material was organized clearly for learning to occur.
Strongly Disagree
1 2 3

3. The content learned from this activity will impact my practice.

Strongly Disagree

$\begin{array}{lllll}1 & 2 & 3 & 4 & 5\end{array}$

4. The activity was presented objectively and free of commercial bias.

Strongly Disagree

$\begin{array}{lllll}1 & 2 & 3 & 4 & 5\end{array}$

To obtain credit, visit Medscape online at http://www.medscapecme.org/journal/jnccn. 